\title{
Herbal prescription Chang'an II repairs intestinal mucosal barrier in rats with post-inflammation irritable bowel syndrome
}

\author{
Feng-yun WANG ${ }^{1, \#}$, Min SU ${ }^{2, \#}$, Yong-qiu ZHENG ${ }^{3}$, Xiao-ge WANG ${ }^{1}$, Nan $\mathrm{KANG}^{1}$, Ting CHEN ${ }^{1}$, En-lin ZHU ${ }^{1}$, \\ Zhao-xiang BIAN ${ }^{4}, *$, Xu-dong TANG ${ }^{1, *}$ \\ ${ }^{1}$ Gastroenterology Department, Xiyuan Hospital, China Academy of Chinese Medical Sciences, Beijing 100091, China; ${ }^{2}$ Xuanwu \\ Traditional Chinese Medical Hospital, Beijing 100000, China; ${ }^{3}$ Research Center, Xiyuan Hospital, China Academy of Chinese Medical \\ Sciences, Beijing 100091, China; ${ }^{4}$ School of Chinese Medicine, Hong Kong Baptist University, Kowloon Tong, China
}

Aim: The herbal prescription Chang'an II is derived from a classical TCM formula Tong-Xie-Yao-Fang for the treatment of liver-qi stagnation and spleen deficiency syndrome of irritable bowel syndrome (IBS). In this study we investigated the effects of Chang'an II on the intestinal mucosal immune barrier in a rat post-inflammation IBS (PI-IBS) model.

Methods: A rat model of PI-IBS was established using a multi-stimulation paradigm including early postnatal sibling deprivation, bondage and intrarectal administration of TNBS. Four weeks after TNBS administration, the rats were treated with Chang'an II (2.85, 5.71 and $11.42 \mathrm{~g} \mathrm{~kg}^{-1} \cdot \mathrm{d}^{-1}$, ig) for $14 \mathrm{~d}$. Intestinal sensitivity was assessed based on the abdominal withdrawal reflex (AWR) scores and fecal water content. Open field test and two-bottle sucrose intake test were used to evaluate the behavioral changes. CD ${ }^{+}$and $C D 8^{+}$ cells were counted and IL-1 $1 \beta$ and IL-4 levels were measured in intestinal mucosa. Transmission electron microscopy was used to evaluate ultrastructural changes of the intestinal mucosal barrier.

Results: PI-IBS model rats showed significantly increased AWR reactivity and fecal water content, and decreased locomotor activity and sucrose intake. Chang'an II treatment not only reduced AWR reactivity and fecal water content, but also suppressed the anxiety and depressive behaviors. Ultrastructural study revealed that the gut mucosal barrier function was severely damaged in PI-IBS model rats, whereas Chang'an II treatment relieved intestinal mucosal inflammation and repaired the gut mucosal barrier. Furthermore, PI-IBS model rats showed a significantly reduced $\mathrm{CD} 4^{+} / \mathrm{CD} 8^{+}$cell ratio in lamina propria and submucosa, and increased IL-1 $\beta$ and reduced IL-4 expression in intestinal mucosa, whereas Chang'an II treatment reversed PI-IBS-induced changes in $\mathrm{CD}^{+} / \mathrm{CD} 8^{+}$cell ratio and expression of IL-1 $\beta$ and IL-4.

Conclusion: Chang'an II treatment protects the intestinal mucosa against PI-IBS through anti-inflammatory, immunomodulatory and anti-anxiety effects.

Keywords: irritable bowel syndrome; Tong-Xie-Yao-Fang; abdominal withdrawal reflex; open field test; two-bottle sucrose intake test; intestinal mucosal barrier; interleukins; T cells

Acta Pharmacologica Sinica (2015) 36: 708-715; doi: 10.1038/aps.2014.170; published online 11 May 2015

\section{Introduction}

Irritable bowel syndrome (IBS) is a chronic gastrointestinal disorder with a reported high prevalence $(7 \%-10 \%)$ in the world population ${ }^{[1]}$. Patients with IBS suffer from recurrent abdominal pain and altered bowel habits. Clinical IBS is not only confined to the colon, but it also extends to other organs and systems. This disease is associated with multiple comor-

\footnotetext{
\# These authors contributed equally to this work.

* To whom correspondence should be addressed.

E-mail txdly@sina.com (Xu-dong TANG);

bzxiang@hkbu.edu.hk (Zhao-xiang BIAN)

Received 2014-09-21 Accepted 2014-12-29
}

bidities such as dyspepsia, interstitial cystitis, fibromyalgia, chronic fatigue, insomnia, headache/migraine and psychiatric disturbances $^{[2]}$.

The herbal prescription Chang' an II is derived from a classical traditional Chinese medicine (TCM) formula, ie, Tong-XieYao-Fang (TXYF), which was one of the first recorded TCMs and has been used to treat the liver-qi stagnation and spleen deficiency syndrome of IBS $^{[3]}$ since the Yuan dynasty. Experimental evidence has shown that the analgesic effect of TXYF is obvious in IBS rats by adjusting the level of 5-hydroxytryptamine (5-HT) and corticotropin releasing factor $(\mathrm{CRF})^{[4]}$. Clinical study results have demonstrated that TXYF has an important role in anti-pain and anti-diarrhea effects on diarrhea- 
irritable bowel syndrome (D-IBS) ${ }^{[5]}$. Recently, researches have also shown that TXYF regulates the levels of cytokines in the colonic mucosa, which could be the possible molecular mechanism for its effect on D-IBS ${ }^{[6]}$. However, the precise mechanism of TXYF action has not become fully clear.

Because the herbal prescription Chang' an II for Shugan and warming the spleen and bowel has been used to treat IBS, an experimental animal model that shares some features with human IBS is needed for the comprehensive study of the neuroimmuno-gastroenterology of IBS and liver-qi stagnation and spleen deficiency. In this study, a post-inflammation irritable bowel syndrome (PI-IBS) rat model was established through complex stimulation with early postnatal sibling deprivation (EPSD), bondage and trinitro-benzene-sulfonic acid (TNBS) administration. In fact, this animal model exhibits symptoms that most closely match those of PI-IBS, such as interstitial cystitis, visceral hypersensitivity and psychiatric disturbances, which were assessed in the present study.

Component contents of Chang'an II are as follows: TXYF (paeoniflorin $0.15 \%$, hesperidin $0.0014 \%$, atractylenolide, radix sapshnikoviae) and ginger carbon. The peony and pericarpium citri reticulatae have been recognized as valuable traditional herbs in TCM and are used in the treatment of inflammatory immune diseases. Modern pharmacological studies have demonstrated that the peony has immunoregulatory effects, anti-inflammatory effects, and anti-anxiety effects ${ }^{[7-9]}$. However, additional studies are required to clarify the protective effects of Chang'an II on the intestinal mucosal barrier in the PI-IBS model. Hence, further investigations may offer new insights into the systemic pharmacological repair mechanisms of Chang'an II. All in all, the aim of the present study was to evaluate the effects of the herbal prescription Chang'an II on the intestinal mucosal immune barrier using a new rat PI-IBS model.

\section{Materials and methods} Animals

Adult pregnant Sprague-Dawley rats (220-230 g), provided by the Animal Facility at the Health Science Center of Peking University (Beijing, China), were housed in the laboratory animal room and maintained at $25 \pm 1^{\circ} \mathrm{C}$ with $65 \% \pm 5 \%$ humidity on a $12 \mathrm{~h} \mathrm{light/dark} \mathrm{cycle} \mathrm{(lights} \mathrm{on} \mathrm{from} \mathrm{7:30} \mathrm{to} \mathrm{19:30).}$ Animals were given food and water ad libitum. Only newborn male rats were used. All experimental protocols described in this study were approved by the Ethics Review Committee for Animal Experimentation of Xiyuan Hospital, China Academy of Chinese Medical Sciences.

\section{Experimental medicine}

The ingredients of Chang' an II were as follows: $18 \mathrm{~g}$ rhizoma atractylodis macrocephalae, $12 \mathrm{~g}$ radix paeoniae alba, $9 \mathrm{~g}$ pericarpium citri reticulatae, $6 \mathrm{~g}$ radix sapshnikoviae and $10 \mathrm{~g}$ ginger carbon, which are clinically used as the daily dose for human.

Chang'an II (brown cream, paeoniflorin $38.35 \mathrm{mg} / \mathrm{g}$ exact and atractylodes $47.76 \mathrm{mg} / \mathrm{g}$ and hesperidin $0.57 \mathrm{mg} / \mathrm{g}$ ) was provided by the Department of Pharmaceutical Preparation of Xiyuan Hospital, according to good manufacturing practices. All herbs were bought from qualified suppliers in China, and high-performance liquid chromatography (HPLC) fingerprint analysis of Chang'an II was performed for quality control of the preparation (see Supplementary Figure 1). Chang' an II was kept at room temperature. The rats were stimulated with TNBS for 3 weeks and then given Chang'an II $(2.85,5.71$, or $11.42 \mathrm{~g} / \mathrm{kg}$ ) intragastrically (ig) once daily for 14 consecutive days (Figure 1). The sham and PI-IBS model groups were given saline. The dosage of Chang' an II used for the present experiment was calculated according to the clinical dosage of the raw materials from TXYF.

The interleukin (IL)-1 $\beta$ enzyme-linked immunosorbent assay (ELISA) kit, IL-4 ELISA kit, mouse CD4 mAb kit and mouse CD8 mAb kit were purchased from R\&D Systems, Inc (ebioscience, San Diego, CA, USA).

\section{Early postnatal sibling deprivation (EPSD)}

Litters were randomly allocated for EPSD treatment at 9:0012:00 AM, from post-natal d 2 (PN2) to PN14. For the normal controls, litters were housed together with their mother. After weaning, the pups were housed together with the same sex and group (4 pups per cage). The pups' physical development was recorded, and body weight was recorded on PN1, PN7, PN14, PN21, PN28, PN42, and PN56. Ear unfolding, eye opening, testis descent and vaginal opening were detected on PN3, PN13, PN23, and PN36, respectively. Maternal care behaviors, including licking/grooming, were observed during PN1-14. From PN30 on, the male adult pups from the EPSD group were randomly divided into the following 4 groups $(n=8)$ : the PI-IBS model group, the PI-IBS+Chang' an II high-dose group $(11.42 \mathrm{~g} / \mathrm{kg}$ ), the PI-IBS+Chang' an II medium-dose group (5.71 $\mathrm{g} / \mathrm{kg})$, and the PI-IBS+Chang' an II low-dose group $(2.85 \mathrm{~g} / \mathrm{kg})$ (Figure 1).

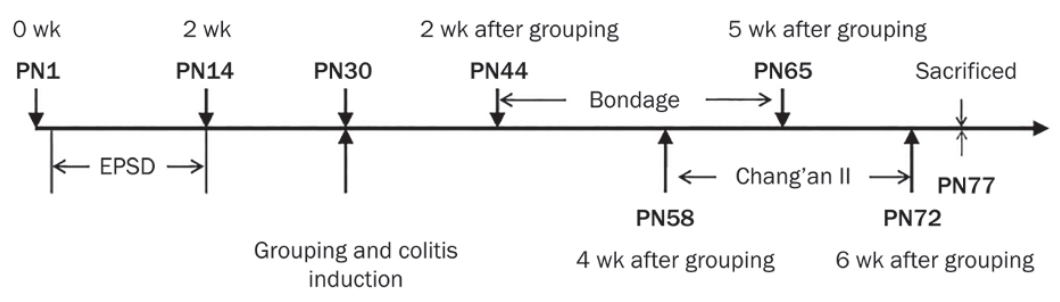

Figure 1. Experimental protocol. 


\section{Induction of colitis using TNBS}

After pentobarbital anesthesia, colitis was induced in the rats according to published methods ${ }^{[10-12]}$ via the intrarectal administration of $0.3 \mathrm{~mL}$ of a solution of TNBS (30 mg) in 50\% ethanol at PN30. The control rats received $0.3 \mathrm{~mL}$ of the $50 \%$ ethanol vehicle. All solutions were delivered via a soft catheter introduced $8 \mathrm{~cm}$ beyond the anus.

\section{Bondage stress}

Bondage stress was imposed on the rats for $3 \mathrm{~h}$ per day (9:0012:00 AM) for 21 continuous days from PN44 (2 weeks after grouping).

\section{Abdominal withdrawal reflex (AWR)}

One day after colitis induction and one day after Chang'an II medication, colorectal distension (CRD) was administered, and the AWR score was quantified for individual rat in all groups. CRD was performed as previously described ${ }^{[13]}$. A latex double-lumen catheter attached to a balloon dilator $(2 \mathrm{~mm}$ in diameter) was used. The balloon was coated with vaseline and inserted into the descending colon with the distal tip 8 $\mathrm{cm}$ from the anus, and CRD was maintained via water injection. The rats were placed in small lucite cubicles and were allowed to wake up and adapt for $15 \mathrm{~min}$. To achieve an accurate measurement, the distention was repeated 3 times. Blind observers measured the AWR responses, which were assessed according to the following scale: 0 , no behavioral response to CRD; 1 , brief head movement followed by immobility; 2 , contraction of the abdominal muscles; 3 , lifting of the abdomen; and 4 , body arching and lifting of pelvic structures. In the present study, CRD was calculated as the quantity of water injected when the AWR score was 3.

\section{Open field test}

Rats were placed individually in the middle of an open field apparatus (height: $40.0 \mathrm{~cm}$; length: $100.0 \mathrm{~cm}$; width: $100.0 \mathrm{~cm}$ ) between 8:00 and 11:00 AM. As described in previous studies $^{[14,15]}, 25$ squares $(20.0 \mathrm{~cm} \times 20.0 \mathrm{~cm})$ were delineated on the floor, and the number of times the rats crossed between squares was counted by two observers (blind to the experimental groups) over a 10-min period. Crossing, as a measure of locomotion, was counted when the rat moved all four legs from one quadrant to another. After each trial, the open field apparatus was carefully cleaned.

\section{Two-bottle sucrose intake test}

To test the effect of Chang'an II on depression, the two-bottle sucrose intake test was performed as described in a previous study ${ }^{[14]}$. Rats were housed singly over the test. The test procedure included three steps. (1) Adaptation: on d 1 after Chang' an II medication, a bottle filled with water and a bottle filled with $2 \%$ sucrose solution were placed in symmetric positions on the same cage wall at 2:00 PM. On d 2 of Chang'an II medication, the locations of the water and sucrose bottles were switched to balance side preference. (2) Deprivation: immediately following adaptation, the sucrose solution was removed, and the rats were deprived of both food and water for $23 \mathrm{~h}$. (3) Test: immediately following deprivation, all animals were given 1-h access to one bottle of a $2 \%$ sucrose solution and one bottle of tap water. At the end of the test, all rats were returned to group housing with ad libitum access to food and water. The volume of the two solutions was used as the index of drinking.

\section{Transmission electron microscopy (TEM)}

TEM was used to evaluate the effect of Chang'an II on the intestinal mucosal barrier at the ultrastructural level. Rats were sacrificed on the 5th day after Chang'an II medication.

Sucrose preference $(\mathrm{SP})=$

$\frac{\text { Sucrose solution consumption }(\mathrm{g})}{\text { Sucrose solution consumption }(\mathrm{g})+\text { water consumption }(\mathrm{g})} \times 100 \%$

Intestinal mucosal fragments were fixed with $2.5 \%$ glutaraldehyde solution overnight at $4{ }^{\circ} \mathrm{C}$ and with $1 \%$ osmic acid for $2 \mathrm{~h}$ followed by 3-time washes with PBS. After the specimens were embedded in an Epon/Araldite mixture and stained with uranyl acetate and lead citrate, the intestinal mucosal barrier was observed under a 1230 type transmission electron microscope (Electron Co, Tokyo, Japan) and photographed.

\section{Measurement of IL-4 and IL-1 $\beta$}

The concentrations of IL- 4 and IL- $1 \beta$ in the supernatants of intestinal mucosa homogenates were measured via ELISA using commercially available kits (R\&D) according to the manufacturer's instructions. The recorded values are presented in $\mathrm{U} / \mathrm{L}$.

\section{Immunohistochemical examination of $\mathrm{CD}^{+}$and $\mathrm{CD}^{+}$cells}

To examine CD4 and CD8 expression, the proximal and distal colon were collected and cut into $4-\mu \mathrm{m}$-thick sections and processed for CD4 and CD8 staining. The number of positive cells was counted in six high-power fields (400x) for each coded slide, and the mean was calculated.

\section{Data quantification and statistical analysis}

All data are presented as the mean \pm SD. Statistical significance was analyzed via one-way analysis of variance (ANOVA) followed by Tukey's test for multiple comparisons. $P<0.05$ was considered statistically significant.

\section{Results}

Effects of Chang'an II on body weight, AWR, and fecal water content in PI-IBS rats

After the TBNS treatment, the rats presented decreased body weight, compared with the control rats (Figure 2). There were no differences in body weight between the model group and the Chang' an II groups (Figure 2A). In comparison to the PIIBS group, water injection in the Chang'an II groups clearly increased at an AWR score of 3 (Figure 2B). The PI-IBS group showed a significant decrease in water injection quantity and at an AWR score of 3, which coincided with higher fecal water content (Figure 2C). These results suggest that the rats treated 

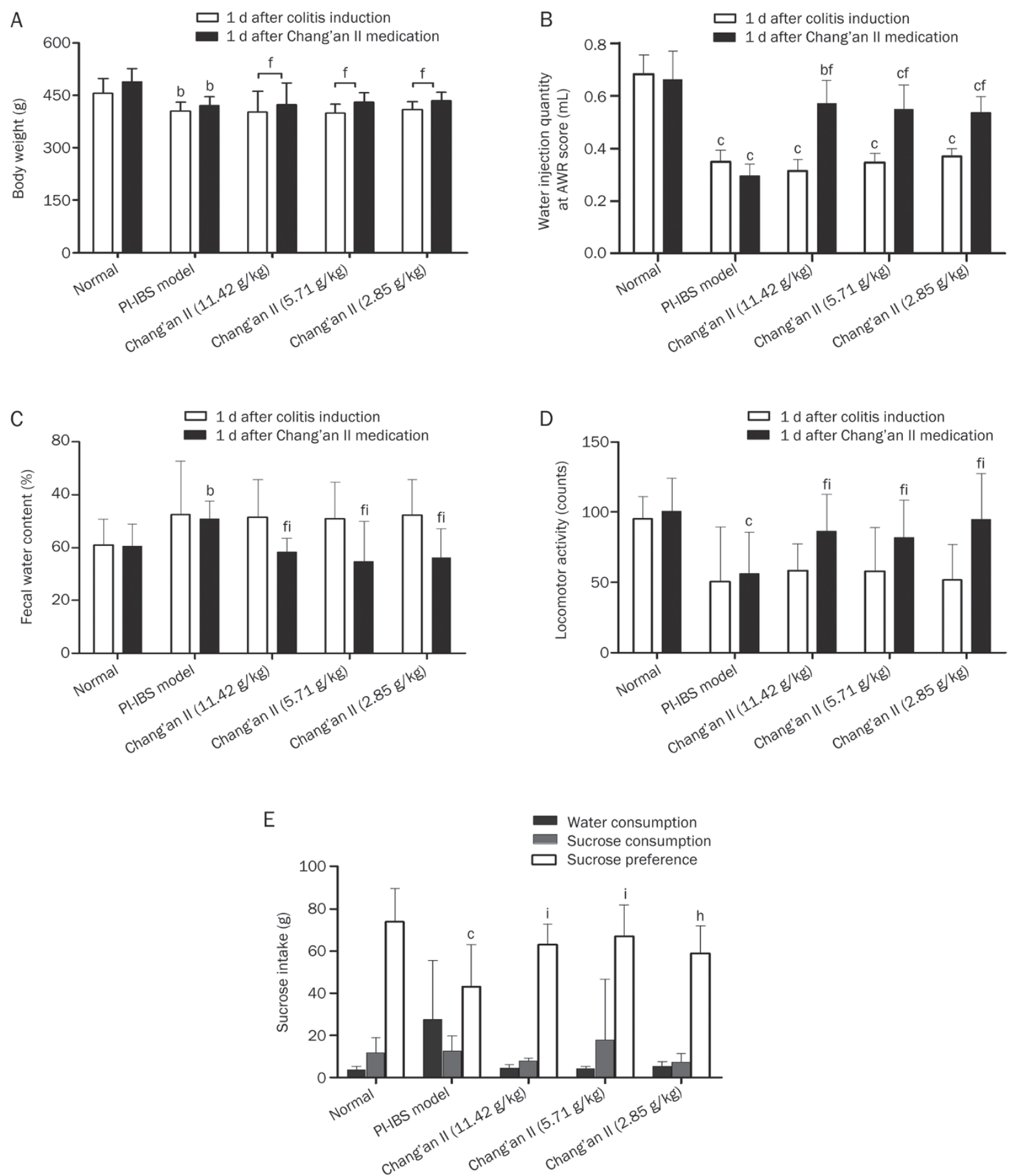

Figure 2. Effects of Chang'an II on body weight, AWR, and fecal water content in PI-IBS rats. (A) Rats' body weight, (B) water injection quantity at an AWR score of 3, (C) fecal water content, (D) locomotor activity and (E) water consumption and sucrose preference in control and Chang'an II treated groups. Mean \pm SD. $n=8$. ${ }^{b} P<0.05,{ }^{c} P<0.01$ vs normal. ${ }^{f} P<0.01$ vs $1 \mathrm{~d}$ after colitis induction. ${ }^{\mathrm{h}} P<0.05,{ }^{\mathrm{i}} P<0.01$ vs model.

with TNBS showed symptoms of PI-IBS with visceral hypersensitivity.

\section{Effects of Chang'an II on locomotor activity (open field test)}

As shown in Figure 2D, ANOVA analysis revealed significant inhibitory effects of EPSD treatment on the crossing times $(P<0.01)$. However, Chang' an II treatment increased the cross- ing times in the open field test $(P<0.05)$, which suggests that Chang' an II reduced the rats' anxiety behaviors.

\section{Effects of Chang'an II on sucrose intake}

Figure 2E shows the volume of water and sucrose consumed during the sucrose preference test. ANOVA analysis revealed that there was a significant decrease in sucrose preference after 


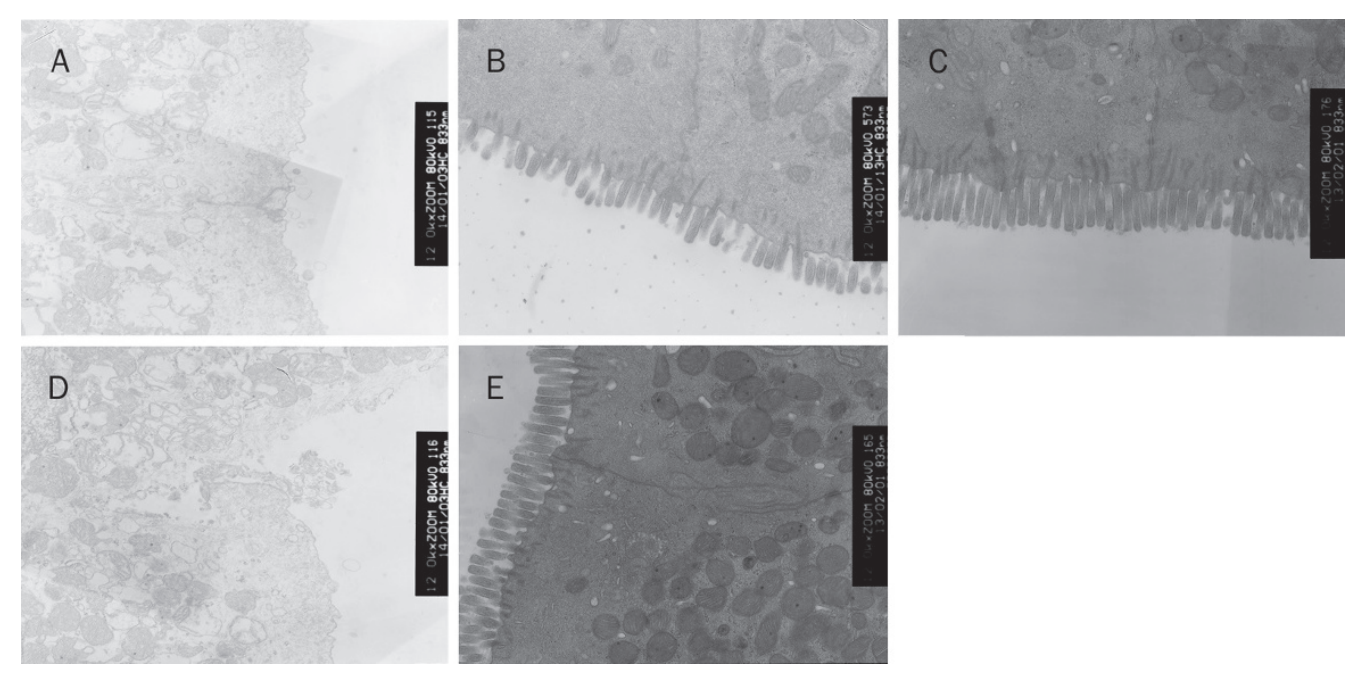

Figure 3. Ultrastructural morphology of the intestinal mucosal barrier. (A) Model group; (B) Chang'an II 11.42 g/kg group; (C) Chang'an II 5.71 g/kg group; (D) Chang'an II $2.85 \mathrm{~g} / \mathrm{kg}$ group; (E) Normal group.

EPSD treatment $(P<0.05)$. However, Chang'an II treatment increased sucrose preference, which suggests that Chang'an II inhibited depression in the rats.

Effects of Chang'an II on the ultrastructural morphology of the intestinal mucosal barrier

The intestinal mucosal barrier in the normal control rats showed integrity mediated by colonic epithelial cells (CECs), whereas CECs in the PI-IBS rats displayed significant edema. Moreover, the PI-IBS rats showed a loss of intestinal mucosal barrier integrity, with pericellular edema and vacuolation and membrane damage. Intracellularly, the nuclei of CECs were swollen, the mitochondria were decreased and swollen with decreasing ridges, and the rough endoplasmic reticulum (RER) was decreased and dilated with degranulation. However, oral administration of Chang'an II (2.85, 5.71, and 11.42 $\mathrm{g} / \mathrm{kg}$ ) repaired the injury mentioned above (Figure 3 ).

\section{Chang'an II prevents colitis-associated influx of inflammatory} cells into the colonic submucosa

As shown in Figures 4, TNBS-induced colitis was associated with increased numbers of $\mathrm{CD}^{+}$cells and decreased $\mathrm{CD} 4^{+}$ cells in the lamina propria and submucosa (Figure 4A and 4B). However, the rats treated with Chang'an II showed significantly lower numbers of $\mathrm{CD}^{+}$cells and higher ratios of $\mathrm{CD}^{+} /$ $\mathrm{CD}^{+}$cells $(P<0.01$, Figure $4 \mathrm{C})$.

To study whether the inflammatory process was affected, we evaluated the concentrations of IL- 4 and IL- $1 \beta$ in the supernatants of intestinal mucosa homogenates. Colitis induction was associated with increased levels of IL-1 $\beta$ and decreased levels of IL-4 $(P<0.01)$. However, the rats treated with Chang'an II showed significantly decreased levels of IL-1 $\beta$ and increased levels of IL-4 compared with the vehicle controls $(P<0.05)$ (Figure 5).

\section{Discussion}

IBS is a common gastrointestinal disorder and is considered to be a functional disease because there appears to be no associated anatomical defect. Stress and psychological factors are thought to have an important role in IBS $^{[16]}$. In addition, there is growing acceptance that acute gastroenteritis may precipitate the development of IBS $^{[17]}$, and recent research has suggested that interactions among the gut, immune system, and nervous system lead to IBS. Therefore, therapeutic options have expanded to relieve bowel movement-related symptoms and pain ${ }^{[18,19]}$. Many patients also seek help from complementary and alternative therapies, primarily Chinese herbal formulas $(\mathrm{CHFs})^{[20]}$.

TCM is commonly used to treat IBS in China, although the results have been shown to be conflicting. In fact, the therapeutic effects of herbal drugs are very difficult to evaluate because of variations in botanical components, the lack of purification and quality control, and the absence of preclinical animal models, and other factors ${ }^{[21,22]}$. In this study, the rat model of PI-IBS, with associated symptoms of visceral hypersensitivity, high fecal water content, anxiety, depression, and slight colitis, was developed through stimulation with EPSD, bondage and TNBS. Together, these symptoms coincided with liver-qi stagnation and spleen deficiency in TCM. After treatment with Chang'an II, the PI-IBS rats presented not only with lower AWR scores and fecal water content but also with a reduction in depression and anxiety, with significantly decreased permeability. In addition to previously identified behavioral determinants, inflammation and immune cells are important in the development of IBS (Figure 4). Our results indicate that Chang'an II induced protection of the intestinal mucosal barrier, which may have been due to a decrease in the $\mathrm{CD}^{+} / \mathrm{CD}^{+}$ratio and an increase in the level of IL-4. Stress is known to enhance responses to subsequent inflammatory 
A $\operatorname{CD4}$
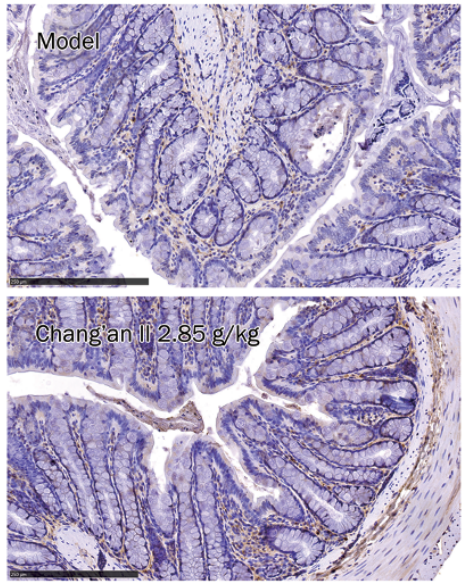

B CD8
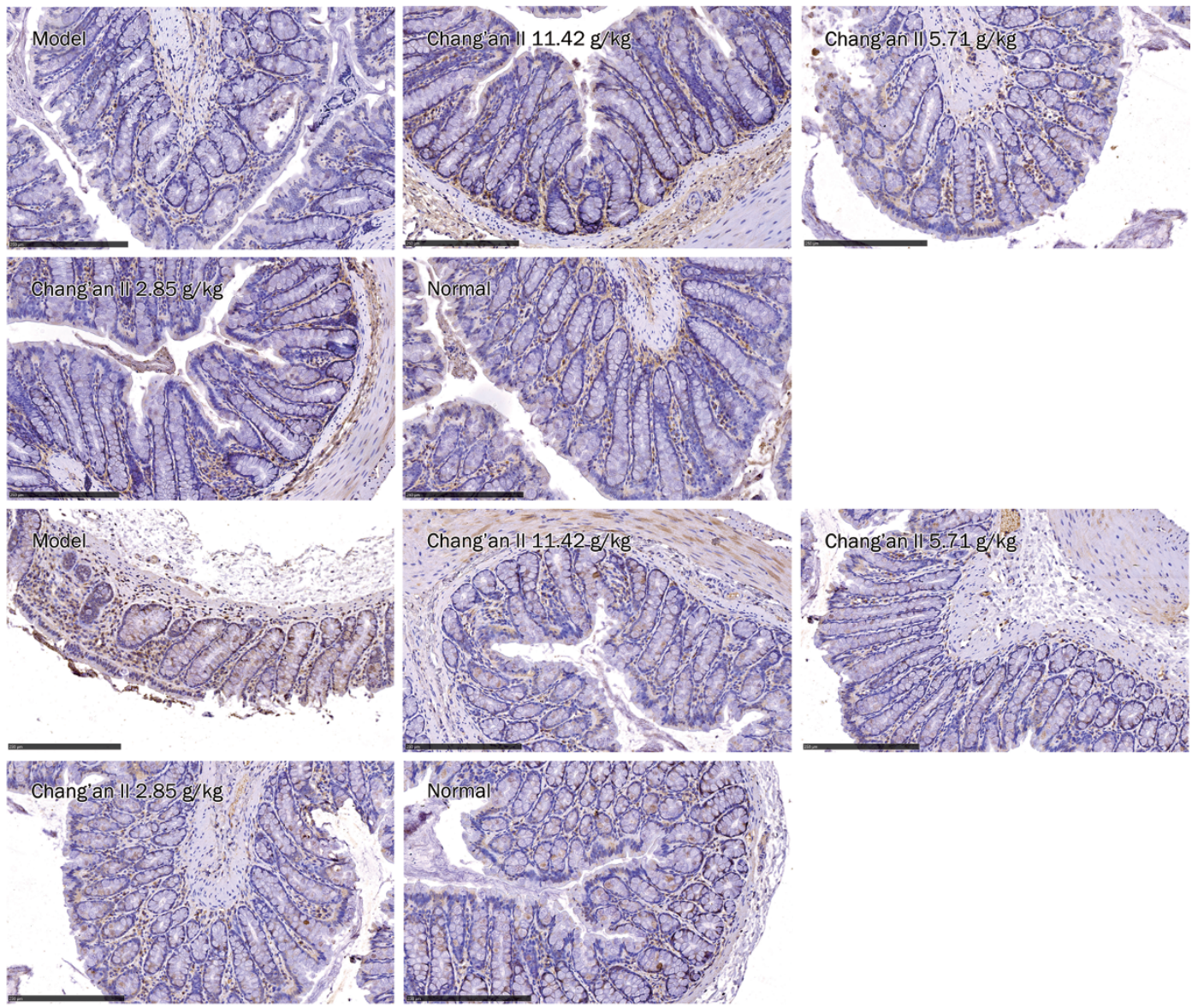

C

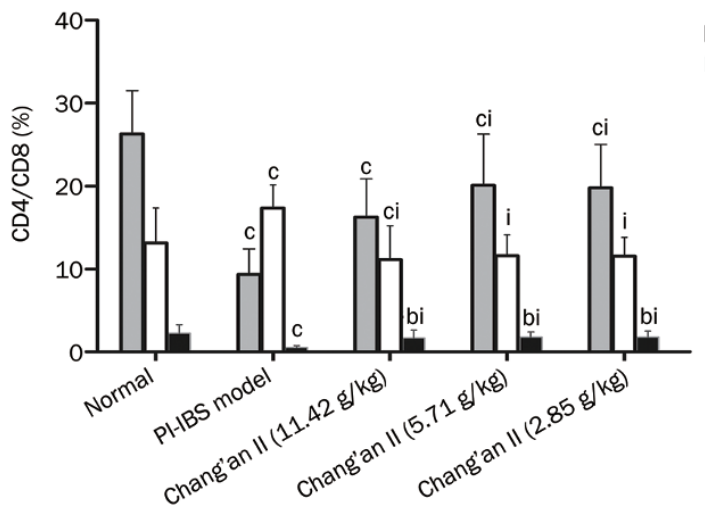

Figure 4. Expression of $\mathrm{CD}^{+}$and $\mathrm{CD} 8^{+} \mathrm{T}$ lymphocytes in the lamina propria and submucosa of the proximal and distal colon. (A and $\mathrm{B}$ ) Immunohistochemical detection for expression of $\mathrm{CD}^{+}$and $\mathrm{CD} 8^{+} \mathrm{T}$ cells. (C) The ratio of $\mathrm{CD} 4^{+} / \mathrm{CD} 8^{+}$cells in control and treated groups. Mean $\pm \mathrm{SD}$. $n=8$. ${ }^{\mathrm{b}} P<0.05,{ }^{\mathrm{c}} P<0.01$ vs normal. ${ }^{\mathrm{i}} P<0.01$ vs model.

stimuli characterized by colonic paracellular permeability due to disruption of tight junctions ${ }^{[23,24]}$. Intestinal permeability can be assessed using multiple techniques. We used electron microscopy in this study, and supporting examples are available in animal-based studies. Our results showed that improvements in intestinal barrier function and intestinal hypersensitivity were closely related to inflammation and stress.
Modern pharmacological studies have demonstrated that atractylodes lactone, the most important component of Chang' an II, provides anti-inflammatory and anti-anxiety effects $^{[1,25]}$, whereas the total glucosides of the peony, another important ingredient of Chang'an II, have shown immunoregulatory effects and anti-inflammatory effects ${ }^{[8,9]}$. Moreover, the mixture of these components has been shown to be superior to the individual components alone. The model 

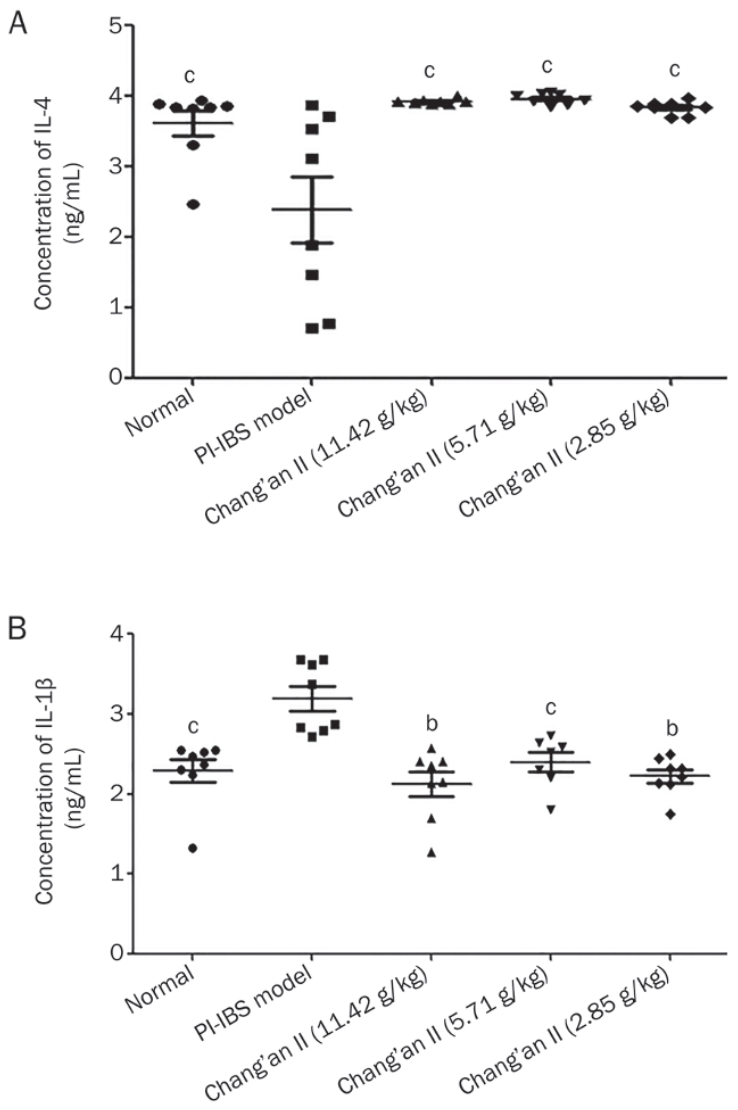

Figure 5. IL-4 and IL-1 $1 \beta$ expression in supernatants of intestinal mucosa homogenates. Data are presented as the mean \pm SD. $n=8$. ${ }^{b} P<0.05$, ${ }^{\mathrm{c}} P<0.01$ vs model.

rats in our experiments showed greater levels of tension, anxiety and other emotions than the animals that received Chang' an II. Our findings support the hypothesis that behavioral factors are important determinants in the development of IBS post-infection.

In conclusion, Chang' an II protected the intestinal mucosa against PI-IBS owing to its anti-inflammatory, immunomodulatory and anti-anxiety effects.

\section{Acknowledgements}

This work was supported by the General Program of National Natural Science Foundation of China (№ 81373580 and 81173209) and the Visiting Scientist Program of China Academy of Chinese Medical Sciences (№ ZZ070801).

\section{Author contribution}

Xu-dong TANG and Zhao-xiang BIAN designed the study; Feng-yun WANG coordinated the experiments and drafted the manuscript; Min SU participated in the design and analysis of the experiment; Xiao-ge WANG carried out the animal study; Nan KANG participated in the experimental design and data analyses; Ting CHEN conducted the extraction of RNA and RT-PCR; En-lin ZHU measured the contents of IL-4 and IL-1 $\beta$ in colonic mucosa using ELISA; and Yong-qiu
ZHENG was involved in discussion of the experiments and drafts of the manuscript. All authors read and approved the final manuscript.

\section{Supplementary information}

Supplementary information is available at Acta Pharmacological Sinica's website.

\section{References}

1 Spiegel BM. The burden of IBS: looking at metrics. Curr Gastroenterol Rep 2009; 11: 265-9.

2 Chang FY. Irritable bowel syndrome: the evolution of multi-dimensional looking and multidisciplinary treatments. World J Gastroenterol 2014; 20: 2499-514.

3 Bian Z, Wu T, Liu L, Miao J, Wong H, Song L, et al. Effectiveness of the Chinese herbal formula TongXieYaoFang for irritable bowel syndrome: a systematic review. J Altern Complement Med 2006; 12: 401-7.

4 Hu XG, Xu D, Zhao Y, Yang XB, Meng J, Shen H. The alleviating pain effect of aqueous extract from tong-xie-yao-fang, on experimental visceral hypersensitivity and it mechanism. Biol Pharm Bull 2009; 32: 1075-9.

5 Hu XG, Xu D, Zhao Y, Yang XB, Meng J, Shen H, et al. The alleviating pain effect of aqueous extract from tong-xie-yao-fang, on experimental visceral hypersensitivity and its mechanism. Biol Pharm Bull 2009; 32: 1075-9.

6 Hu X, Zhang X, Han B, Bei W. The inhibitory effect of tongxieyaofang on rats with post infectious irritable bowel syndrome through regulating colonic par-2 receptor. BMC Complement Altern Med 2013; 13: 246.

7 Zheng YQ, Wei W, Zhu L, Liu JX. Effects and mechanisms of Paeoniflorin, a bioactive glucoside from paeony root, on adjuvant arthritis in rats. Inflammation Res 2007; 56: 1-7.

8 Zheng YQ, Wei W. Total glucosides of paeony suppresses adjuvant arthritis in rats and intervenes cytokine-signaling between different types of synoviocytes. Int Immunopharmacol 2005; 5: 1560-73.

9 Hu X, Zhang X, Han B, Bei W. The inhibitory effect of tongxieyaofang on rats with post infectious irritable bowel syndrome through regulating colonic par-2 receptor. BMC Complement Altern Med 2013; 13: 246.

10 Wallace JL, Keenan CM. An orally active inhibitor of leukotriene synthesis accelerates healing in a rat model of colitis. Am J Physilo Gastrointest Liver Physiol 1990; 258: 527-34.

11 Moreels TG, Nieuwendijk RJ, De Man JG, De Winter BY, Herman AG, Van Marck EA. Concurrent infection with Schistosoma mansoni attenuates inflammation induced changes in colonic morphology, cytokine levels, and smooth moscle contractility of trinitrobenzene sulphonic acid induced colitis in rats. Gut 2004; 53: 99-107.

12 Stein J, Ries J, Barrett KE. Disruption of intestinal barrier function associated with experimental colitis: possible role of mast cells. Am J Physiol 1998; 274: G203-9.

13 Al-Chaer ED, Kawasaki M, Pasricha PJ. A new model of chronic visceral hypersensitivity in adult rats induced by colon irritation during postnatal development. Gastroenterology 2000; 119: 1276-85.

14 Li YQ, Wang XY, Zhai HF, Zheng YQ, Zhang XY, Kosten T, et al. Effects of early postnatal sibling deprivation on anxiety and vulnerability to cocaine in offspring rats. Psychopharmacology (Berl) 2008; 199: 245-53.

15 Rex A, Voigt JP, Voits M, Fink H. Pharmacological evaluation of a modified open-field test sensitive to anxiolytic drugs. Pharmacol Biochem Behav 1998; 59: 677-83.

16 El-Salhy M, Gundersen D, Gilja OH, Hatlebakk JG, Hausken T. Is irritable bowel syndrome an organic disorder? World J Gastroenterol 2014; 20: 384-400. 
17 Rodríguez LA, Ruigómez A. Increased risk of irritable bowel syndrome after bacterial gastroenteritis: cohort study. BMJ 1999; 318: 565-6.

18 Camilleri M. Review article: new receptor targets for medical therapy in irritable bowel syndrome. Aliment Pharmacol Ther 2010; 31: $35-$ 46.

19 Maneerattanaporn M, Chang L, Chey WD. Emerging pharmacological therapies for the irritable bowel syndrome. Gastroenterol Clin North Am 2011; 40: 223-43.

20 Hussain Z, Quigley EM. Systematic review: complementary and alternative medicine in the irritable bowel syndrome. Aliment Pharmacol Ther 2006; 23: 465-71.

21 Brandt LJ, Chey WD, Foxx-Orenstein AE, Schiller LR, Schoenfeld PS, et al. An evidence-based position statement on the management of irritable bowel syndrome. Am J Gastroenterol 2009; 104 supple 1:
S1-35.

22 Bensoussan A, Talley NJ, Hing M, Menzies R, Guo A, Ngu M. Treatment of irritable bowel syndrome with Chinese herbal medicine: a randomized controlled trial. JAMA 1998; 280: 1585-9.

23 Frank CF, Hostetter MK. Cleavage of E-cadherin: a mechanism for disruption of the intestinal epithelial barrier by Candida albicans. Transl Res 2007; 149: 211-22.

24 Zhang Q, Li Q, Wang C, Liu X, Li N, Li J. Enteropathogenic Escherichia coli changes distribution of occludin and ZO-1 in tight junction membrane microdomains in vivo. Microb Pathog 2010; 48: 28-34.

25 Singhuber J, Baburin I, Kählig H, Urban E, Kopp B, Hering S. GABA(A) receptor modulators from Chinese herbal medicines traditionally applied against insomnia and anxiety. Phytomedicine 2012; 19: 334-40. 